\title{
MiR-1-3p inhibits cell proliferation and invasion by regulating BDNF-TrkB signaling pathway in bladder cancer
}

\author{
L. GAO ${ }^{1}$, P. YAN ${ }^{2}$, F.F. GUO ${ }^{3}$, H.J. LIU ${ }^{3}$, Z.F. ZHAO ${ }^{3, *}$ \\ ${ }^{1}$ Department of Lithotriptic Center, Linyi People's Hospital, Linyi, Shandong, 276000, China; ${ }^{2}$ Department of Rheumatology, Linyi People's Hos- \\ pital, Linyi, Shandong, 276000, China; ${ }^{3}$ Department of Urinary Surgery, Linyi People’s Hospital, Linyi, Shandong, 276000, China
}

*Correspondence: mozhaohui_xy@126.com

Received November 28, 2016 / Accepted June 9, 2017

\begin{abstract}
Recent studies have confirmed the existence of BDNF and tropomyosin-related kinase B (TrkB) in normal and cancerous urothelium. However, the corresponding mechanisms and upstream signal pathways of BDNF/TrkB have not been fully discovered. This study aimed to investigate the effects of miR-1-3p on bladder cancer (BC) by regulating BDNF-TrkB signal pathway. The expression of miR-1-3p and BDNF in BC tissues and cell lines were detected by Cancer Genome Atlas (TCGA) microarray analysis, RT-qPCR and western blot. Cell transfection was done using Lipofectamine 2000. Then cell viability, proliferation, migration and apoptosis were measured by MTT, colony formation assay, Transwell assay and flow cytometry, respectively. The relationship between miR-1-3p and BDNF was confirmed by luciferase reporter gene assay. MiR-1-3p was significantly down-regulated in BC tissues and cell lines, while BDNF was significantly up-regulated compared to normal samples. MiR-1-3p targeted BDNF and suppressed its expression. Transfections of miR-1-3p mimics and BDNF siRNAs can suppress BC cell proliferation, invasion and induce cell apoptosis. In addition, miR-1-3p can inhibit phosphorylation of the TrkB by regulating BDNF.

In conclusion, MiR-1-3p has significant effects on viability, proliferation, invasion and apoptosis of BC cells by regulating BDNF-TrkB pathway.
\end{abstract}

Key words: MiR-1-3p, BDNF, TrkB, bladder cancer

Bladder cancer (BC) is a fatal disease due to its high mortality and a large amount of populations is afflicted by this disease [1]. The most common type of BC is transitional cell carcinoma (TCC), which is usually derived from urothelial epithelium [2]; other types of BC include squamous cell carcinoma and adenocarcinoma [3]. The selection of BC therapies depends on the cancer differentiation, which may vary from individual to individual and several therapies have been developed, including surgery, radiation therapy, intravesical chemotherapy and systematic chemotherapy [4]. Nevertheless, the overall prognosis of $\mathrm{BC}$ patients has limited improvement [5]. Therefore, discovering novel anti-cancer therapies to improve the survival status of BC patients is a critical issue.

MicroRNAs (miRNAs) are a group of non-coding short RNAs that negatively affect gene expression by binding to the 3' untranslated region (3' UTR) of mRNAs [6]. MiRNAs are considered to be either oncogenic factors or tumor suppressors according to the base-pairing roles to their target genes, thereby enhancing the degradation of mRNAs and inhibit- ing the post-transcriptional translation. For instance, miR124 potentially suppresses the growth of bladder cancer by targeting CDK4 3'UTR in vitro and in vivo [7], and miR-144 exhibits a potential role in inhibiting cancer proliferation by targeting EZH2 3'UTR [8]. Moreover, miR-1 is another potential tumor suppressor in a variety of cancers and such a suppressive function may also exist in BC cell lines [9]. However, the corresponding roles and functions of miR-1 family have not been identified thoroughly in BC.

Brain-derived neurotrophic factor (BDNF), a pro-neuronal factor, exerts its effects on regulating neural maturation and differentiation by binding to its preferred receptor tropomyosin-related kinase B (TrkB) [10]. Also, recent studies have confirmed that both BDNF and TrkB exist in normal urothelium and tumors. These studies concluded that BDNF mediates activation of the $\operatorname{TrkB}$ which is a survival signal for TCC cells, implying its oncogenic role in BC [11]. However, the corresponding mechanisms and upstream signal pathways of BDNF/TrkB have not been fully discovered yet. 
Our study demonstrated that miR-1-3p was down-regulated in $\mathrm{BC}$ cell lines, implying its suppressive effect on $\mathrm{BC}$ and such an effect is potentially achieved by the activation of BDNF-pTrkB signal pathway. Therefore, this study aimed to unravel the effects of miR-1-3p on BC by regulating BDNFTrkB signal pathway. Our study revealed a potential role of miR1-3p and BDNF-pTrkB in proliferation, invasion and apoptosis of BC by analyzing the corresponding effects in BC cells.

\section{Materials and methods}

TCGA microarray analysis. The Cancer Genome Atlas (TCGA) microarray was used to analyze the expression of miRNA and mRNA in samples from cancerous bladder and normal bladder tissues. The inclusion criterion is Fold Change value $>2(\mathrm{p}<0.05)$.

Cell lines and cell culture. In this study, we used the human bladder transitional carcinoma cell lines UM-UC-3 and T24, human bladder carcinoma cell lines 5637 and TCCSUP, and human epithelial ureter immortalized cell line SV-HUC-1 (all cell lines were purchased from Shanghai Institutes for Biological Sciences, Chinese Academy of Cell Resource Center). All cell lines were cultured in Dulbecco's modified eagle medium (DMEM) containing 10\% fetal bovine serum (FBS, Hyclone Co., South Logan, UT, USA) in a $5 \% \mathrm{CO}_{2}$ incubator at $37^{\circ} \mathrm{C}$.

Cells transfection. We transfected the negative control, miRNA-1-3p mimics and BDNF siRNAs (purchased from Ribobiobiological Technology Co., Ltd., Guangzhou, China) into UM-UC-3 cells by Lipofectamine 2000 (Invitrogen Corporation, Carlsbad, CA, USA) according to the instructions. Cells were then divided to 4 groups consisting of con group (UMUC-3 cells without transfection), NC group (UM-UC-3 cells transfected with Negative Controls), miR-1-3p group (UMUC-3 cells transfected with miR-1-3p mimics), and siBDNF group (UM-UC-3 cells transfected with BDNF siRNAs).

Real-time quantitative PCR (RT-qPCR). Total RNA was extracted by Trizol reagent (Invitrogen, Inc., Carlsbad, CA, USA) according to the instructions. CDNA was synthesized using mRNA and miRNA reverse transcription kit (Takara Biotechnology Co., Ltd., Dalian, China). Finally, we used the cDNA as a template for $\mathrm{RT}$-qPCR reactions using PCR kit (Takara Biotechnology Co., Ltd.). U6 and GAPDH were used as internal controls for miRNA and mRNA respectively. The relative expression of miRNA and mRNA was calculated using the $2^{-D D C}$ method. Primer sequences are shown in Table 1.
Western blot assay. Total protein was separated from samples, and the protein concentration was determined using bicinchoninic acid assay (BCA) reagent kit (Beyotime, China). After protein electrophoresis, the membranes were blocked with $5 \%$ skimmed milk at room temperature for 4 hours and then incubated for 1 hour at $37^{\circ} \mathrm{C}$ with primary antibodies (anti-GAPDH, anti-BDNF, anti-TrkB and anti-pTrkB rabbit polyclonal antibodies, Sigma-Aldrich St. Louis, MO, USA) diluted by confining liquid at 1:1000, followed by 4 wash times using Tris Buffered Saline Tween (TBST). The membranes were then incubated at $37^{\circ} \mathrm{C}$ for 1 hour with HPR-conjugated secondary antibodies (Sigma-Aldrich St. Louis, MO, USA) diluted to 1:5000 with TBST and washed 4 times in TBST again. Immunoreactive bands were visualized with an enhanced chemiluminescence (WesternBrightTM ECL kit, Advansta Corporation, Menlo Park, CA, USA), according to the manufacturer's instructions. Blots were scanned and optical density evaluated using ImageJ software (1.45s) (NIH, Bethesda, MD, USA).

MTT (3-[4, 5-dimethylthiazol-2-yl]-2, 5 diphenyltetrazolium bromide) assay. After transfection for $48 \mathrm{~h}$, the UMUC-3 cells were seeded in 96 -well plates $\left(5 \times 10^{3}\right.$ cells/well). After $24 \mathrm{~h}, 48 \mathrm{~h}, 72 \mathrm{~h}, 96 \mathrm{~h}$ and $120 \mathrm{~h}$ of cultivation, MTT solution (Sigma-Aldrich, St. Louis, MO, USA) was added ( $5 \mathrm{mg} / \mathrm{mL}, 20 \mu \mathrm{L} /$ well $)$. After another 4 -hour cultivation, the supernatant was discarded and DMSO solution $(150 \mu \mathrm{L} /$ well $)$ was added. After a 10-minute oscillation in dark, the optical density (OD) of cells in each well was measured in the microplate reader (EL405, Bio-Tek Instruments, Winooski, VT, USA) at $490 \mathrm{~nm}$.

Colony formation assay. The transfected cells were digested by pancreatic enzymes and made into a single-cell suspension. Then the cells were seeded in 6 -well plates $\left(5 \times 10^{2}\right.$ cells/ well) and placed in the incubator. After 14-day cultivation, cells were fixed with $4 \%$ paraformaldehyde solution at room temperature for 15 minutes and then they were stained with $0.2 \%$ crystal violet. The number of cell clones was counted under an optical microscope.

Transwell invasion assay. Invasion analysis was conducted using $8 \mu \mathrm{m}$-pore transwell chambers (purchased from Corning, Incorporated (Corning, NY, USA). 48 hours after transfection, $200 \mu \mathrm{l}$ of non-serum cell suspension containing around $2 \times 10^{4}$ cells was seeded into upper chambers coated with $50 \mu \mathrm{l}$ matrigel (1:4; BD BiosciencesSan Jose, CA, USA), while the lower chambers contained $500 \mu \mathrm{D}$ DMEM with $10 \%$ FBS. Cells were then cultured for another 24 hours and those

Table 1. Primer sequences of miR-1-3p and BDNF for implementation in RT-qPCR.

\begin{tabular}{lll}
\hline Gene & Forward primer & Reverse primer \\
\hline MiRNA-1-3p & 5'-UGGAAUGUAAAGAAGUAUGUAU-3' & \\
BDNF & 5'-AACCATAAGGACGCGGACTT-3' & 5'-TGCAGTCTTTTTATCTGCCG-3' \\
U6 & 5'-CTCGCTTCGGCAGCACA-3' & 5'-AACGCTTCACGAATTTGCGT-3' \\
GAPDH & 5'-TGAAGGTCGGAGTCAACGG-3' & 5'-CCTGGAAGATGGTGATGGG-3' \\
\hline
\end{tabular}

GAPDH: phosphoglyceraldehyde dehydrogenase, RT-qPCR: real time-polymerase chain reaction 
failing to pass through the filter membrane were removed from the membrane. Finally, the cells that passed through the membrane were dyed with $0.1 \%$ crystal violet and counted from 5 random perspectives under a microscope. All experiments in each group were done in triplicates.

Flow cytometry. For cell cycle assay, $3 \times 10^{5}$ cells in each group were collected and fixed with $70 \%$ cold ethanol overnight at $4{ }^{\circ} \mathrm{C}$. Cells were then washed with PBS 3 times and treated with $100 \mathrm{mg} / \mathrm{ml}$ RNase A for $0.5 \mathrm{~h}$. Subsequently, the cells were treated with $50 \mathrm{mg} / \mathrm{ml}$ propidium iodide (PI) for $0.5 \mathrm{~h}$ in the dark. DNA distribution was measured using a FACScan flow cytometer (BD Biosciences) thereafter. Cell apoptosis was calculated after staining the cells with Annexin V-FITC/PI Apoptosis Detection Kit (BD Biosciences).

Dual luciferase reporter gene assay. The wild type BDNF 3'UTR sequence (position 1322-1329) was amplified by using RT-qPCR and inserted into pmirGLO luciferase carrier (Promega Corporation, USA), named BDNF wt. As for the mutated type of plasmid, BDNF mut, the complementary sequences for miR-1-3p at BDNF 3'UTR were mutated using positioning mutation method. Luciferase carrier was directly transfected into UM-UC3 cells combined with miRNA mimics or negative controls, respectively. 48 hours after transfection, DLR dual luciferase reporter assay system (Promega Corporation, USA) was conducted to measure the luciferase activity in cells. The luciferase activity was defined as the ratio of Firefly luciferase to Renilla luciferase. Both wild type and mutated BDNF 3'UTR sequences were synthesized by Sangon Biotech Company (Shanghai, China). There are 3 binding sites of BDNF 3'UTR of miR-1-3p. We haven't tried the other binding sites, but we chose the position 1322-1329 of BDNF 3' UTR in this case, because this particular sequence has the highest context score and the lowest PCT (probability of conserved targeting) according to TargetScan $7.0 \mathrm{Hu}-$ man algorithm. Indeed, study on the other binding sites of BDNF 3'UTR are warranted, especially given that Varendi et al. found that miR-1 could regulate either short or long BDNF 3'UTR isoforms [12].

Statistical methods. All data analyses were performed using SPSS 18.0 statistical software (IBM, Chicago, Illinois, USA). The measurement data were shown as a mean \pm standard deviation $(\bar{x} \pm s)$. T-test was used to compare the difference between any two groups if data were normally distributed. One-way ANOVA was used to compare differences between multiple groups. It would be considered statistically different when $\mathrm{p}<0.05$.

\section{Results}

The expression of miR-1-3p and BDNF in BC tissues and cells. TCGA data was used to analyze the expression of miRNA and mRNA in bladder cancer samples. As shown in Figure 1, BC tissues had significantly lower miR-1-3p expression but higher BDNF expression than normal tissues $(\mathrm{p}<0.05)$.
The expressions of miR-1-3p and BDNF in human bladder cancer cell lines UM-UC-3, T24, 5637, TCCSUP and human ureter epithelial immortalized cell line SV-HUC-1 were detected using RT-qPCR and western blot. When compared with SV-HUC-1, all human bladder cancer cells had significantly lower miR-1-3p levels but higher BDNF levels $(p<0.05$, Figure 2). UM-UC-3 showed the lowest miR-1-3p levels but the highest BDNF levels, therefore we chose this cell line for further experiments.

Targeting relationship between BDNF and miR-1-3p. As predicted by miRDB database, there was a targeted binding site for miR-1-3p in BDNF 3' UTR (Figure 3A). To confirm the targeting relationship, we conducted the dual luciferase reporter gene assay in cell line UM-UC-3. As expected, cells transfected with wild type BDNF (BDNF wt) and miR-1-3p mimics had significantly lower luciferase intensity than the NC group ( $<<0.05)$, whereas cells transfected with mutated BDNF (BDNF mut) and miR-1-3p mimics had no significant difference when compared to the NC group ( $p>0.05$, Figure $3 \mathrm{~B}$ ). The results indicated that miR-1-3p directly regulates BDNF expression by targeting BDNF 3'UTR.

Western blot results revealed that the expression level of BDNF in miR-1-3p group was significantly lower than in the con group and NC group, which is shown in Figure 3C, indicating that the up-regulation of miR-1-3p could suppress the expression of BDNF.

MiR-1-3p and BDNF affected cell viability and proliferation. Cell viability was detected by MTT assay. As shown in Figure 4A, cells transfected with miR-1-3p mimics and BDNF siRNAs had a significantly less active cell viability than in the con group and NC group $48 \mathrm{~h}, 96 \mathrm{~h}$ and $120 \mathrm{~h}$ after the transfection $(p<0.05)$, whereas no significant difference was found between the con and NC groups ( $p>0.05)$.

Then colony formation assay was used to detect cell proliferation. As shown in Figure 4B, no significant difference was found in the number of cell clones between the con and $\mathrm{NC}$ groups $(\mathrm{p}>0.05)$. However, the number of cell clones in miR-1-3p group and siBDNF group significantly decreased compared to con and $\mathrm{NC}$ groups $(\mathrm{p}<0.05)$, suggesting that the proliferation of UM-UC-3 cells was inhibited.

MiR-1-3p and BDNF affected cell invasion. The invasion of cells in different groups was detected by Transwell assay. As shown in Figure 4C, the number of invaded cells in miR1-3p group and siBDNF group was obviously smaller compared with that of the con group and NC group, while no significant difference was found between con group and NC group. We can conclude that the proliferation and invasion of UM-UC-3 cells was reduced by the transfection of miR-1-3p mimics or BDNF siRNAs.

MiR-1-3p and BDNF affected cell cycle and apoptosis. As shown in Figure 5A, when compared with the con group and NC group, the proportion of G0/G1 phase cells was much bigger while the $S$ and $\mathrm{G} 2 / \mathrm{M}$ phase number of cells was much smaller in the miR-1-3p group and siBDNF group $(\mathrm{p}<0.05)$. And the cell apoptosis in miR-1-3p group and siBDNF group 


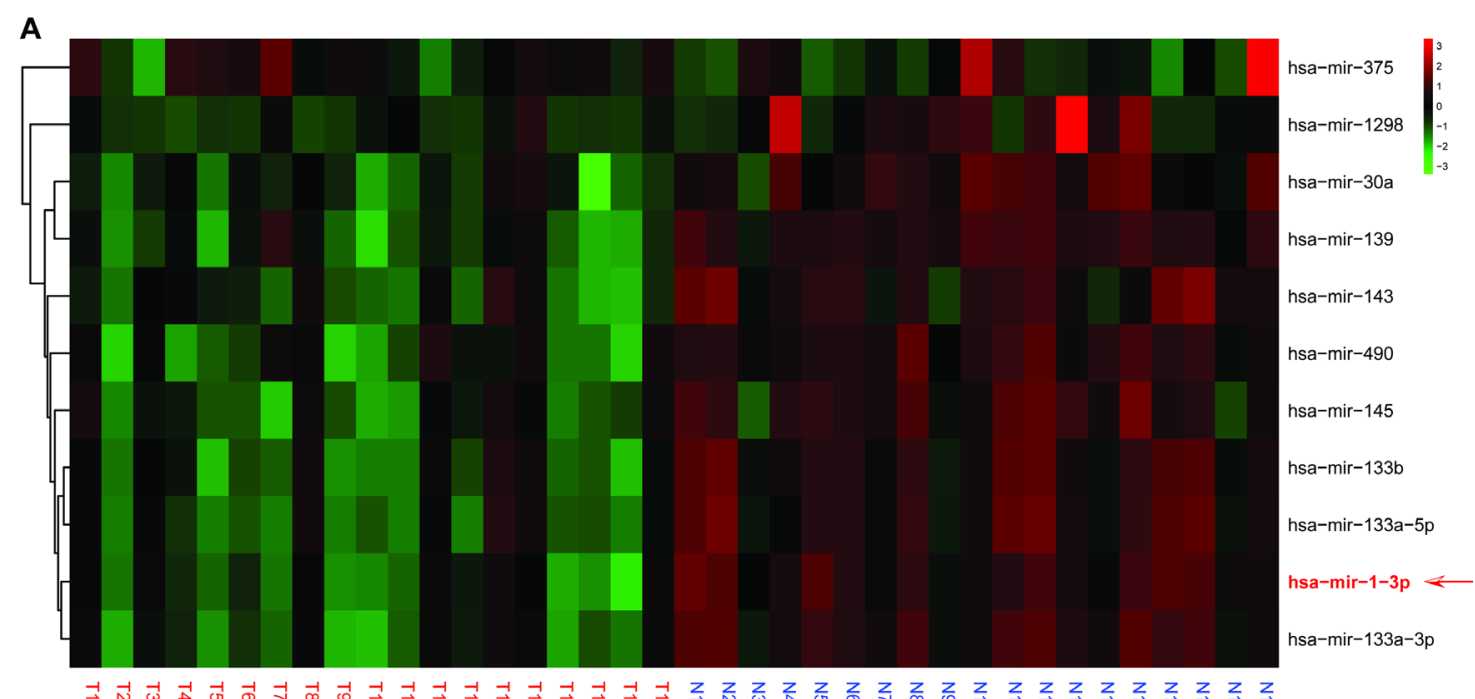

$\mathbf{B}$

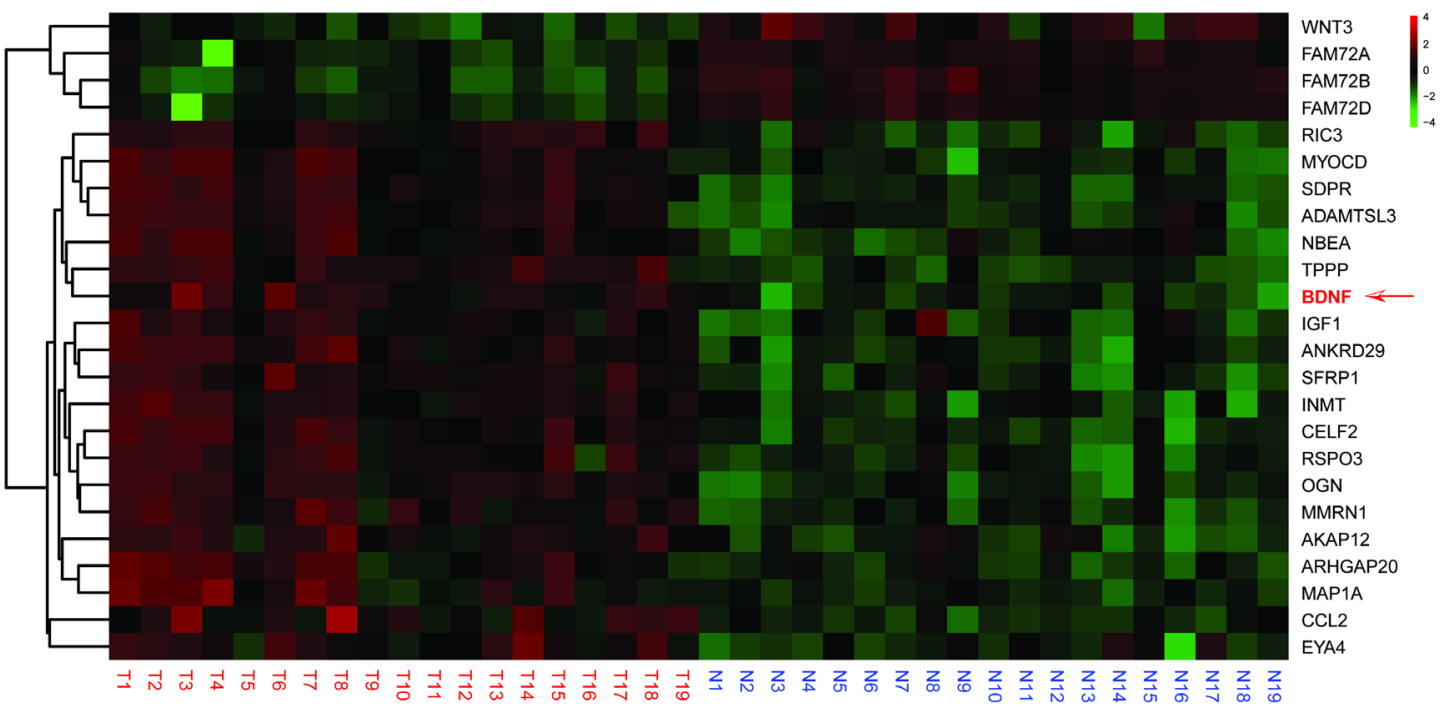

Figure 1. The results of TCGA data analyses. A MiRNA expression difference in normal and diseased patients. Hsa-mir-1-3p was shown to be underexpressed in patients with cancer. B Differential expression of genes in normal and diseased patients. BDNF was shown to be up-regulated in patients with cancer but down-regulated in healthy people. TCGA: The Cancer Genome Atlas.
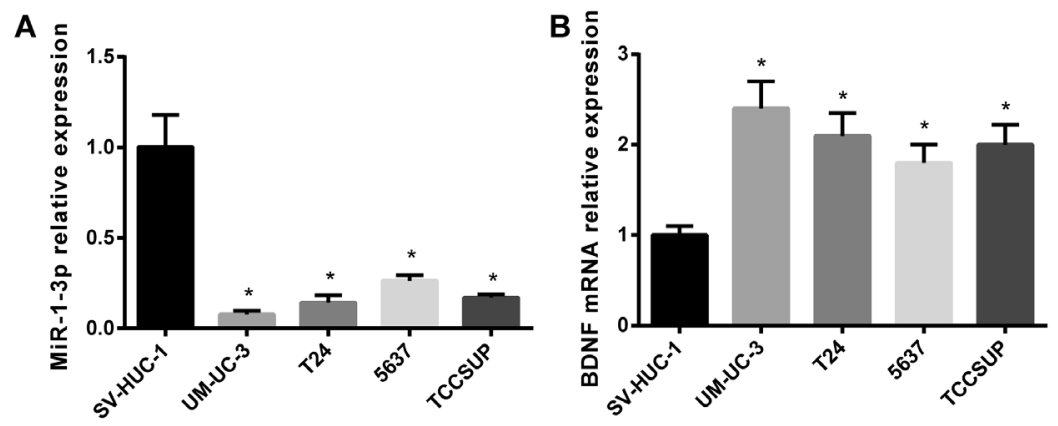

C

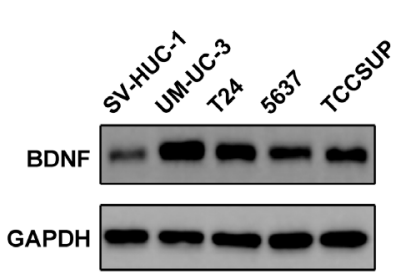

Figure 2. The expression of miR-1-3p and BDNF in BC cell lines. A The RT-qPCR results of miR-1-3p in 5 cell lines. B The RT-qPCR results of BDNF mRNA in 5 cell lines. C The Western Blot results of BDNF protein in 5 cell lines. Data are presented as mean \pm SD for three independent experiments. ${ }^{*} \mathrm{p}<0.05$ versus $\mathrm{SV}-\mathrm{HUC}-1$ group. 

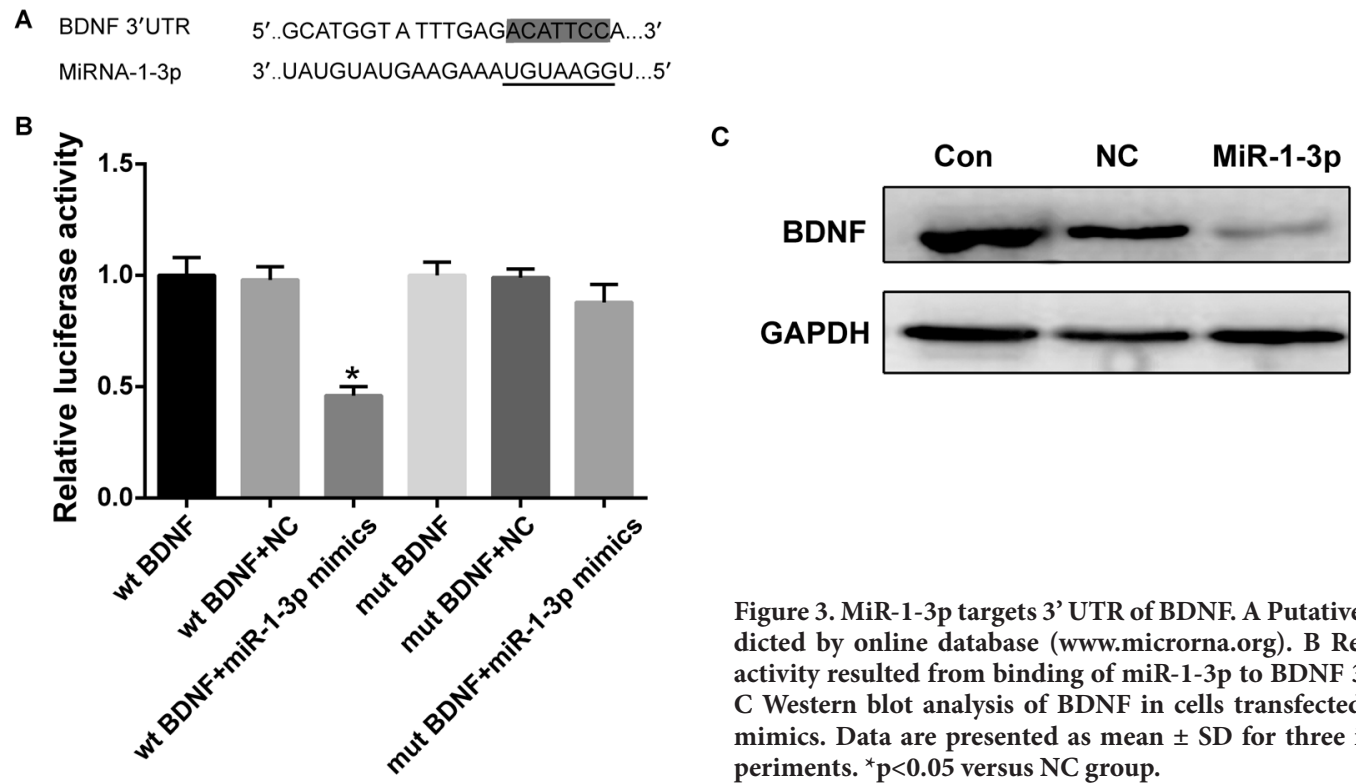

Figure 3. MiR-1-3p targets 3' UTR of BDNF. A Putative target sites predicted by online database (www.microrna.org). B Relative luciferase activity resulted from binding of miR-1-3p to BDNF 3' UTR reporter. C Western blot analysis of BDNF in cells transfected with miR-1-3p mimics. Data are presented as mean \pm SD for three independent experiments. ${ }^{*} \mathrm{p}<0.05$ versus $\mathrm{NC}$ group.
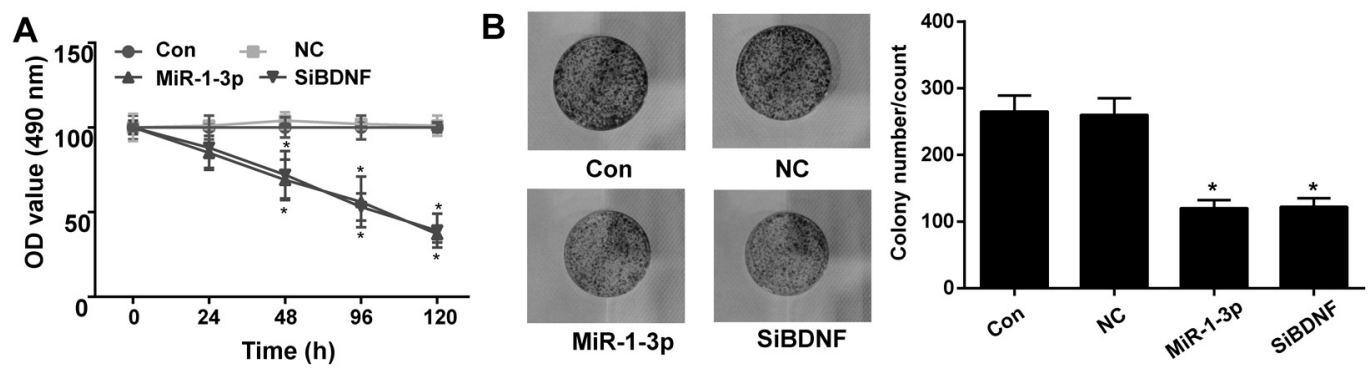

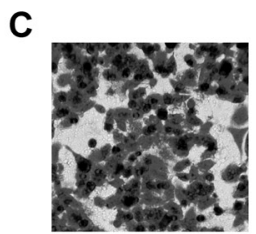

Con

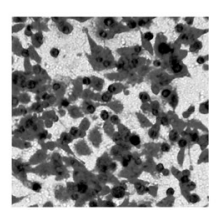

NC

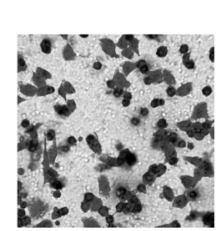

MiR-1-3p

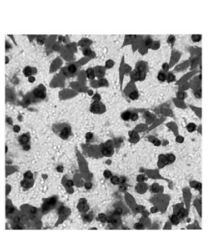

SiBDNF

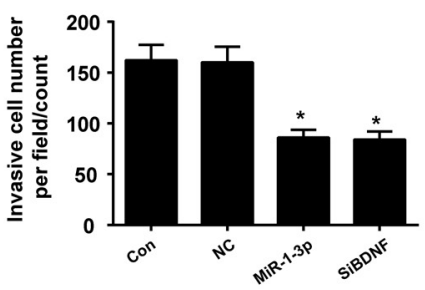

Figure 4. Cell viability, proliferation and invasion results. A MTT assay results demonstrating the effects of miR-1-3p and siBDNF on cell viability. ${ }^{*} \mathrm{p}<\mathbf{0 . 0 5}$ vs. con group. B Colony formation assay results demonstrating the effects of miR-1-3p and siBDNF on cell proliferation. C Transwell assay results showing the effects of miR-1-3p and siBDNF on cell invasion. Data are presented as mean \pm SD for three independent experiments. ${ }^{\star} p<0.05$, compared with the Con group.

significantly increased compared to the con group and $\mathrm{NC}$ group (Figure $5 \mathrm{~B}, \mathrm{p}<0.05$ ). There was no significant difference between con group and NC group (Figure $5 \mathrm{~A}-\mathrm{B}, \mathrm{p}>0.05$ ).

The effect of BDNF suppression on TrkB expression. The results in Figure 6 show the protein expression levels of TrkB and pTrkB in UM-UC-3 cells detected by western blot. Transfection of miR-1-3p mimics or BDNF siRNAs had no obvious effect on the expression of TrkB, but remarkably suppressed the expression of $\mathrm{p} T \mathrm{rkB}$, compared with con group and NC group. The ratio of $\mathrm{p} \operatorname{TrkB} / \operatorname{TrkB}$ decreased, indicat- ing that $\mathrm{BDNF} / \mathrm{TrkB}$ signaling pathway was suppressed in miR-1-3p group and siBDNF group.

\section{Discussion}

Mounting evidence shows that the expression of specific miRNAs is altered in $\mathrm{BC}$, leading to the dysregulation of expression of oncogenes or tumor suppressor genes. For example, miR-194 inhibits proliferation and invasion of cancer cells like a tumor suppressor via repressing RAP2B, playing 

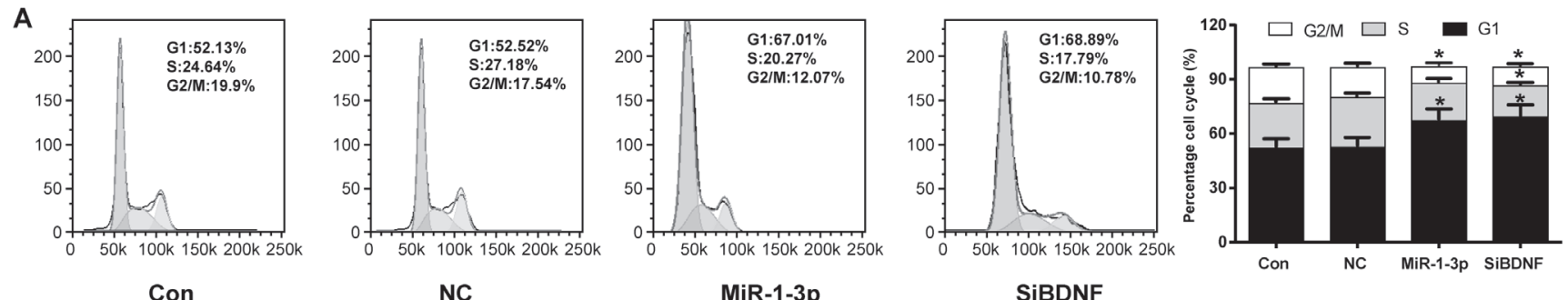

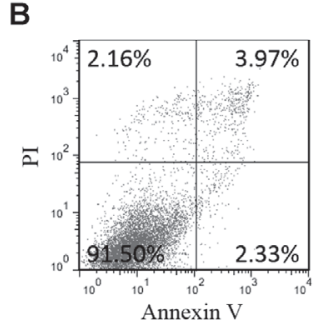

Con

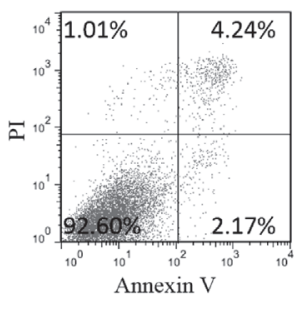

NC

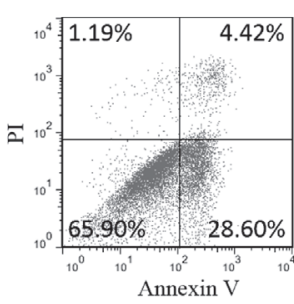

MiR-1-3p
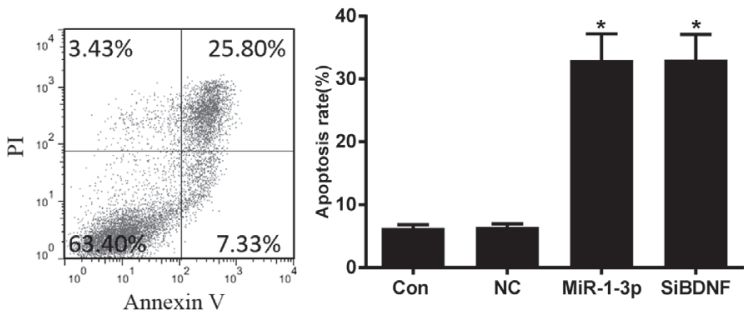

SiBDNF

Figure 5. Cell cycle and cell apoptosis were detected by flow cytometry. A Cell cycle results showing that miR-1-3p and siBDNF significantly prolonged the G0/G1 stage and reduced the S stage. B Cell apoptosis results demonstrating that miR-1-3p and siBDNF substantially promoted cell death. Data are presented as mean $\pm \mathrm{SD}$ for three independent experiments. ${ }^{*} \mathrm{p}<0.05$ versus Con group.

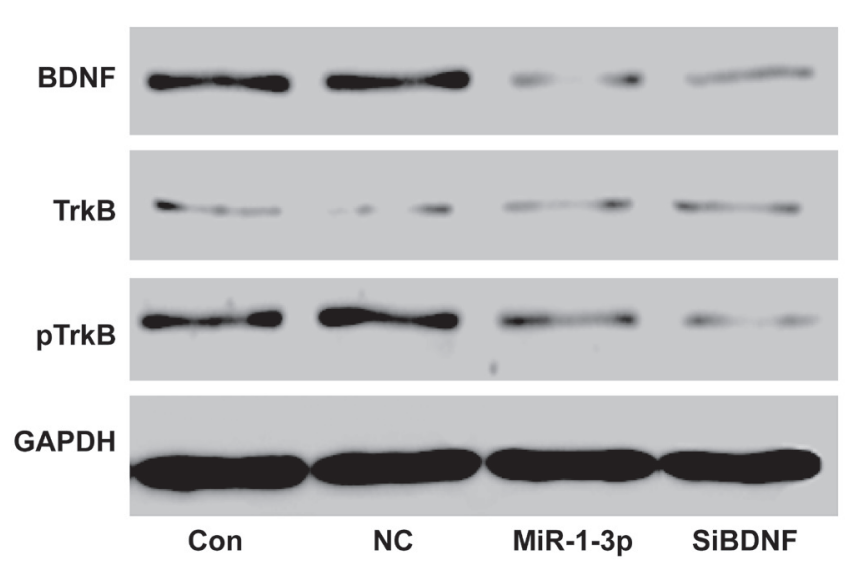

Figure 6. Western blot (WB) analysis of BDNF, TrkB and pTrkB in cells transfected with miR-1-3p mimics and BDNF siRNAs.

a crucial role in the regulation of bladder cancer progression [13]. MiR-96 was significantly expressed in both human BC tissues and cell lines, which reduced the expression of $\mathrm{CD}$ KN1A, functioning as an onco-miRNA [14]. These studies show that different miRNAs may play different roles in BC with different underlying molecular mechanisms. In the present study, we demonstrated that miR-1-3p was downregulated in human $\mathrm{BC}$ cell line UM-UC-3, and the restoration of miR-1-3p expression significantly suppressed the proliferation and invasion of $\mathrm{BC}$ through inhibiting the $\mathrm{BDNF} /$ TrkB pathway, suggesting that miR-1-3p is a potential therapeutic target for BC.

MiR-1 was confirmed to directly regulate BDNF expression in $\mathrm{C} 2 \mathrm{C} 12$ myotubes to maintain differentiation by binding to its 3'UTR [12]. However, whether miR-1 could regulate endogenous BDNF expression remains to be explored. Nonetheless, investigation of the miR-1 has been reported in different cancers. It was found that miR-1 was down-regulated in gastric cancer tissues, and its down regulation played a key role in tumorigenesis, progression, invasion and metastasis [15]. MiR-1 was also found to directly target MET, cyclin D1 and CDK4, which were involved in the hepatocyte growth factor/MET signaling pathway, and suppressed the growth of esophageal squamous carcinoma in vivo and in vitro [16]. In nasopharyngeal carcinoma, its tumor-suppression effects were mediated mainly by repressing K-ras expression, but were inhibited by LMP1 which is an Epstein-Barr virus-associated protein [17]. For its predominant down-regulation in the majority of examined tumors, miR-1 is often classified as a tumor suppressor, having the potential to diminish tumor development [18]. Our study has identified that miR-1-3p is down-regulated in BC by RT-qPCR, suggesting the possibility that miR-1-3p may also play a tumor suppressive role. Our results further indicate that the overexpression of miR-1-3p could inhibit proliferation, invasion but induce cell apoptosis. All these results support the tumor suppressive role of miR-1-3p in BC.

Out of many potential target genes of miR-1-3p predicted by miRDB database, BDNF was selected for further study by previous study and DNA microarrays analysis. BDNF is a member of neurotrophic factors involved in neuronal survival by binding to $\operatorname{TrkB}[19]$. It was demonstrated that BDNF and TrkB are up-regulated in BC especially in TCC [20], and the results indicate that the appearance of BDNF's binding to TrkB could be a survival signal for TCC cells [11]. It was also found that BDNF was overexpressed, which then promoted the propagation of lung cancer cells [21], colorectal cancer cells [22] and ovarian cancer cells [23], showing its causal 
role in different cancers. Consistently, in our study, we also identified that BDNF was upregulated at both protein and mRNA levels in BC cells. Furthermore, when the expression of BDNF was inhibited in UM-UC-3 cells by BDNF siRNA transfection, the inhibition of proliferation, reduction of invasion and higher apoptosis rate were also observed, suggesting that BDNF may be an important factor for BC proliferation and invasion. Together, these results indicate that BDNF plays an opposite role to miR-1-3p in BC cells.

To explore the specific relationship between miR-1-3p and BDNF, and to understand the underlying mechanisms involved in the suppressive processes initiated by miR-1-3p, some more researches have been done. We found that the up-regulation of miR-1-3p could effectively inhibit the expression of BDNF, demonstrating that BDNF was negatively regulated by miR-1-3p. Moreover, luciferase reporter assay confirmed the target relationship between miR-1-3p and BDNF, indicating a negatively modulated relationship. It is the first time that the relationship between them was reported. Besides, we found that $\mathrm{pTrkB}$ level significantly decreased when cells were transfected with miR-1-3p mimics or BDNF siRNAs, meaning that BDNF-TrkB signaling pathway was activated by BDNF, but suppressed by miR-1-3p.

There are still some molecular mechanisms underlying relationship between $\mathrm{pTrkB}$ and $\mathrm{BC}$ cells that we have not quite understood. Besides, there are other target genes of miR-1-3p, therefore studies on the relationship between these genes and miR-1-3p can be useful. Similarly, miR-1-3p is not the only miRNA targeting BDNF, therefore research on the relationship of other miRNAs and BDNF may also be necessary.

In summary, we have demonstrated that miR-1-3p, a down-regulated miRNA in $\mathrm{BC}$, serves as a tumor suppressor. We also demonstrated that miR-1-3p inhibits BC cell proliferation and invasion by modulating BDNF/TrkB pathway. After all, our study reveales a novel mechanism of miR-1-3p in regulating $\mathrm{BC}$ cells, which provides new insights into therapeutic strategies for human BC.

\section{References}

[1] TORRE LA, BRAY F, SIEGEL RL, FERLAY J, LORTETTIEULENT J ET AL. Global cancer statistics, 2012. CA Cancer J Clin 2015; 65: 87-108. doi: 10.3322/caac.21262

[2] CROCKETT DG, WAGNER DG, HOLMANG S, JOHANSSON SL, LYNCH HT. Upper urinary tract carcinoma in Lynch syndrome cases. J Urol 2011; 185: 1627-1630. doi: 10.1016/j.juro.2010.12.102

[3] TAVORA F, EPSTEIN JI. Bladder cancer, pathological classification and staging. BJU Int 2008; 102: 1216-1220. doi: 10.1111/j.1464-410X.2008.07962

[4] DONAT SM. Integrating perioperative chemotherapy into the treatment of muscle-invasive bladder cancer: strategy versus reality. J Natl Compr Canc Netw 2009; 7: 40-47.
[5] WANG CC, CHIANG YM, KUO PL, CHANG JK, HSU YL. Norsolorinic acid inhibits proliferation of T24 human bladder cancer cells by arresting the cell cycle at the G0/G1 phase and inducing a Fas/membrane-bound Fas ligand-mediated apoptotic pathway. Clin Exp Pharmacol Physiol 2008; 35: 1301-1308. doi: 10.1111/j.1440-1681.2008.05007.x

[6] NILSEN TW. Mechanisms of microRNA-mediated gene regulation in animal cells. Trends Genet 2007; 23: 243-249.

[7] ZHANG T, WANG J, ZHAI X, LI H, LI C et al. MiR-124 retards bladder cancer growth by directly targeting CDK4. Acta Biochim Biophys Sin (Shanghai) 2014; 46: 1072-1079. doi: 10.1093/abbs/gmu105

[8] GUO Y, YING L, TIAN Y, YANG P, ZHU Y et al. miR-144 downregulation increases bladder cancer cell proliferation by targeting EZH2 and regulating Wnt signaling. FEBS J 2016; 280: 4531-4538. doi: 10.1111/febs. 12417

[9] YOSHINO H, CHIYOMARU T, ENOKIDA H, KAWAKAMI $\mathrm{K}$, TATARANO $\mathrm{S}$ et al. The tumour-suppressive function of miR-1 and miR-133a targeting TAGLN2 in bladder cancer. Br J Cancer 2011; 104: 808-818. doi: 10.1038/bjc.2011.23

[10] LONG J, JIANG C, LIU B, FANG S, KUANG M. MicroRNA$15 a-5 p$ suppresses cancer proliferation and division in human hepatocellular carcinoma by targeting BDNF. Tumour Biol 2016; 37: 5821-5828. doi: 10.1007/s13277-015-4427-6

[11] HUANG YT, LAI PC, WU CC, HSU SH, CHENG CC et al. BDNF mediated TrkB activation is a survival signal for transitional cell carcinoma cells. Int J Oncol 2010; 36: 1469-1476.

[12] VARENDI K, KUMAR A, HARMA MA, ANDRESSOO JO. miR-1, miR-10b, miR-155, and miR-191 are novel regulators of BDNF. Cell Mol Life Sci 2014; 71: 4443-4456. doi: 10.1007/s00018-014-1628-x

[13] ZHANG M, ZHUANG Q, CUI L. MiR-194 inhibits cell proliferation and invasion via repression of RAP2B in bladder cancer. Biomed Pharmacother 2016; 80: 268-275. doi: 10.1016/j.biopha.2016.03.026

[14] ALBRETSEN CS. [An adolescent narcotic addict treated in her own family group]. Tidsskr Nor Laegeforen 1972; 92: 2349-2351.

[15] XIE M, DART DA, OWEN S, WEN X, JI J et al. Insights into roles of the miR-1, -133 and -206 family in gastric cancer (Review). Oncol Rep 2016; 36: 1191-1198. doi: 10.3892/ or.2016.4908

[16] JIANG S, ZHAO C, YANG X, LI X, PAN Q et al. miR-1 suppresses the growth of esophageal squamous cell carcinoma in vivo and in vitro through the downregulation of MET, cyclin D1 and CDK4 expression. Int J Mol Med 2016; 38: 113-122. doi: $10.3892 / \mathrm{ijmm} .2016 .2619$

[17] CHEN X, SHI J, ZHONG J, HUANG Z, LUO X et al. miR-1, regulated by LMP1, suppresses tumour growth and metastasis by targeting K-ras in nasopharyngeal carcinoma. Int J Exp Pathol 2016; 96: 427-432. doi: 10.1111/iep.12162

[18] WEISS M, BRANDENBURG LO, BURCHARDT M, STOPE MB. MicroRNA-1 properties in cancer regulatory networks and tumor biology. Crit Rev Oncol Hematol 2016; 104: 7177. doi: 10.1016/j.critrevonc.2016.05.014

[19] HUANG EJ, REICHARDT LF. Neurotrophins: roles in neuronal development and function. Annu Rev Neurosci 2001; 24: $677-736$. 
[20] LAI PC, CHIU TH, HUANG YT. Overexpression of BDNF and TrkB in human bladder cancer specimens. Oncol Rep 2010; 24: 1265-1270.

[21] ZHANG SY, HUI LP, LI CY, GAO J, CUI ZS et al. More expression of BDNF associates with lung squamous cell carcinoma and is critical to the proliferation and invasion of lung cancer cells. BMC Cancer 2016; 16: 171. doi: 10.1186/ s12885-016-2218-0
[22] TANAKA K, OKUGAWA Y, TOIYAMA Y, INOUE Y, SAIGUSA $S$ et al. Brain-derived neurotrophic factor (BDNF)induced tropomyosin-related kinase B (Trk B) signaling is a potential therapeutic target for peritoneal carcinomatosis arising from colorectal cancer. PLoS One 2014; 9: e96410. doi: 10.1371/journal.pone.0096410

[23] CORNELIO DB, DE FARIAS CB, PRUSCH DS, HEINEN TE, DOS SANTOS RP et al. Influence of GRPR and BDNF/ TrkB signaling on the viability of breast and gynecologic cancer cells. Mol Clin Oncol 2013; 1: 148-152. 